\title{
Turbidimetric method for evaluation of photocatalytic activities of suspended fine particles
}

This article was published in the following Dove Press journal:

Nanotechnology, Science and Applications

30 September 2010

Number of times this article has been viewed

\author{
Hideki Aoyagi' \\ Katsumi Yabusaki ${ }^{2}$ \\ 'Life Science and Bioengineering, \\ Graduate School of Life and \\ Environmental Sciences, University \\ of Tsukuba, Tsukuba, Ibaraki, Japan; \\ ${ }^{2}$ Electronics and Optics Research \\ Laboratory, Kowa Ltd, Chofugaoka, \\ Chofu City, Tokyo, Japan
}

Correspondence: Hideki Aoyagi Life Science and Bioengineering, Graduate School of Life and Environmental Sciences, University of Tsukuba, Tsukuba, Ibaraki 305-8572, Japan Tel +8 I 2985372 I 2

Fax +8I 298534605

Email aoyagi@sakura.cc.tsukuba.ac.jp
Abstract: A spectrophotometer with special cuvette was developed for evaluating the photocatalytic activities of suspended fine particles. The spectrophotometer can continuously irradiate UV light using LED to the sample solution, and changes in the absorbance at $664 \mathrm{~nm}$ during photocatalytic degradation of methylene blue (MB) were monitored continuously. From the onset of MB degradation, the absorbance decreased and reached a steady value at the end of the reaction. This process was expressed by first order kinetics and the photocatalytic activities of various fine particles could be evaluated quantitatively based on the reaction rate constant $(k)$. The effect of photocatalysis using various $\mathrm{TiO}_{2}$ fine particles on the physiological activities of Euglena gracilis was related with $k$ value.

Keywords: photocatalyst, fine nano sized particles, specialized spectrophotometer, Euglena gracilis, rate constant

\section{Introduction}

The use of nano sized fine particles is of increasing importance because they are suitable for manifold applications in the fields of electronics, ${ }^{1}$ sensing, ${ }^{2}$ catalysis,${ }^{3}$ and medical, ${ }^{4}$ etc. Some types of particles (for example $\mathrm{TiO}_{2}$ ) show photocatalytic activities. Many kinds of photocatalytic fine particles with various activities are commercially produced and widely used for various applications. They are used as photocatalysts in environmental decontamination of air, soil, and water, ${ }^{5-7}$ pigments, ${ }^{8}$ and as cosmetic additives. ${ }^{9}$ Large quantities of the fine particles are discharged to the environment intentionally or unintentionally in the course of their production, use, and disposal. These will inevitably lead to pollution of both the biotic and abiotic components of the environment. Reactive oxygen species (ROS) produced by photocatalysis cause inactivation of various organisms. ${ }^{10-13}$ Studies on the potential effects of these photocatalysts on human health and on the environment are therefore very important. ${ }^{13,14}$ In order to assess the potential risks of photocatalytic fine particles or to efficiently utilize them in various fields, it is very necessary to develop methods for quantitative evaluation of their photocatalytic activities. Many kinds of fine particles with various photocatalytic activities are commercially produced but there are few reports of their relative photocatalytic activities. ${ }^{15}$

In this study, an apparatus was developed for quantitative monitoring of photocatalytic reaction of nano fine particles based on spectrophotometry. With this apparatus, it was possible to evaluate the photocatalytic activities of various fine particles on the basis of the reaction rate constant $(k)$ in first order kinetic. The effect of photocatalysis using $\mathrm{TiO}_{2}$ 
fine particles, which measured $k$ values, on the physiological activities of Euglena gracilis was also investigated.

\section{Materials and methods Chemicals}

Titanium oxide anatase form [ANA] (100\% anatase crystalline form, average particle size $=50 \mathrm{~nm}$, Wako Pure Chemical Industries, Japan), titanium oxide rutile form [RU] (100\% rutile crystalline form, average particle size $=100 \mathrm{~nm}$, Wako) titanium oxide amorphous [AM] (average diameter $=40 \mathrm{~nm}$, Wako); AP25 (20\% rutile $80 \%$ anatase crystalline form, average particle size $=40 \mathrm{~nm}$, Japan Aerosil Co, Tokyo, Japan), STS-01 (photocatalytic sol, average particle size $=7$ nm, Ishihara Sangyo Kaisha, Ltd, Osaka, Japan), and STS-02 (photocatalytic sol, average particle size $=7 \mathrm{~nm}$, Ishihara Sangyo) were used in this study. Methylene blue (MB) and Daigo's artificial seawater SP (in mg per liter: $\mathrm{MgCl}_{2} \cdot 6 \mathrm{H}_{2} \mathrm{O}, 9$, 474: $\mathrm{CaCl}_{2} \cdot 2 \mathrm{H}_{2} \mathrm{O}, 1,326: \mathrm{Na}_{2} \mathrm{SO}_{4}, 3,505$ : $\mathrm{KCl}$, 597: $\mathrm{NaHCO}_{3}, 171: \mathrm{KBr}, 85: \mathrm{Na}_{2} \mathrm{~B}_{4} \mathrm{O}_{7} \cdot 10 \mathrm{H}_{2} \mathrm{O}, 34$ : $\mathrm{SrCl}_{2}$, 12: NaF, 3: LiCl, 1: KI, 0.07: $\mathrm{CoCl}_{2} \cdot 6 \mathrm{H}_{2} \mathrm{O}, 0.0002$ : $\mathrm{AlCl}_{3} \cdot 6 \mathrm{H}_{2} \mathrm{O}, 0.008: \mathrm{FeCl}_{3} \cdot 6 \mathrm{H}_{2} \mathrm{O}, 0.005: \mathrm{Na}_{2} \mathrm{WO}_{4} \cdot 2 \mathrm{H}_{2} \mathrm{O}$, 0.002: $\left(\mathrm{NH}_{4}\right)_{6} \mathrm{Mo}_{7} \mathrm{O}_{24} \cdot 4 \mathrm{H}_{2} \mathrm{O}, 0.02: \mathrm{MnCl}_{2} \cdot 4 \mathrm{H}_{2} \mathrm{O}, 0.0008$ : $\mathrm{NaCl}, 20,747)$ were purchased from Wako.

\section{Cultivation of Euglena gracilis}

E. gracilis IAM E-6 (strain Z), obtained from the algal collection of the Institute of Applied Microbiology, University of Tokyo, Japan was used in this study as a model micro-algae. E. gracilis produces antioxidant such as alpha-tocopherol. Modified Hutner medium was used for the cultivation as described previously. ${ }^{16}$ The medium was sterilized by autoclaving at $121^{\circ} \mathrm{C}$ for $15 \mathrm{~min}$. To prevent deactivation by heat, filter-sterilized vitamin B12 was added to the medium after sterilization. Cultivation was carried out by inoculating E. gracilis into a $500 \mathrm{~mL}$ Erlenmeyer flask containing $100 \mathrm{~mL}$ medium and the flask was then incubated under illumination by black light (National Electric, Tokyo) on rotary shaker $(120 \mathrm{rpm})$ at $25^{\circ} \mathrm{C}$. The distance between the flask and the black light was adjusted to give a light intensity of $1.0 \mathrm{~mW} / \mathrm{cm}^{2}$ at the surface of the flask. The ANA, AP25, or $\mathrm{RU}(100 \mathrm{mg} / \mathrm{L})$ was added to the E. gracilis cell culture.

\section{Analytical methods}

The alpha-tocopherol was extracted from the cell according to the methods. ${ }^{17}$ E. gracilis cells harvested by centrifugation at $1,000 \times g$ for 5 min were washed with $\mathrm{N}_{2}$ saturated distilled water before the addition of $0.1 \mathrm{~mL}$ of $50 \mathrm{mM}$ L-ascorbic acid and extracted with $3.75 \mathrm{~mL}$ of chloroform-methanol
$(1: 2 \mathrm{v} / \mathrm{v})$ by shaking vigorously for $20 \mathrm{~min}$ in $\mathrm{N}_{2}$. The mixture was centrifuged at $1,000 \times g$ for $5 \mathrm{~min}$ to remove cell debris. The extraction was repeated two times. The cell debris was extracted with $4.75 \mathrm{~mL}$ of chloroform-methanol-water $(1: 2: 0.8 \mathrm{v} / \mathrm{v})$. An equal volume of distilled water was added to combined extracts and mixed gently. The chloroform layer was separated from the water-methanol layer after cooling on ice and centrifuged at $1,000 \times g$ for $5 \mathrm{~min}$, and evaporated to dryness at room temperature under a stream of $\mathrm{N}_{2}$ gas to obtain the lipid fraction (the alpha-tocopherol content of the cells was determined from the fraction). Vitamin E homolog kit for biochemistry and analysis (Eisai, Tokyo, Japan) was used for the preparation of the calibration curve. The alpha-tocopherol was measured using high-performance liquid chromatography equipped with an Intelligent Spectrofluorometer 821-FP (Nihon Bunko, Tokyo, Japan) and packed silica gel column (150A-5, $4.6 \times 250 \mathrm{~mm}$, GL Science, Tokyo, Japan). The fluorescent lamp was used for the detection of alpha-tocopherol. The wavelengths were $\mathrm{Ex}=295 \mathrm{~nm}$ and $\mathrm{Em}=340 \mathrm{~nm}$. A mixture of $n$-hexane, 1,4-dioxane, and ethanol (97.6:2.0:0.4 v/v) was used as the elute at a flow rate of $1.5 \mathrm{~mL} / \mathrm{min}$. The column temperature was $40^{\circ} \mathrm{C}$. Dry cell weight was determined according to the method described previously. ${ }^{16}$ The total chlorophyll $(\mathrm{a}+\mathrm{b})$ concentration was determined according to the method ${ }^{18}$ using N, N'-dimethylformamide (DMF). Cells were extracted with DMF overnight. The cells were removed by centrifugation $(1,000 \times g$ for $5 \mathrm{~min})$, and the absorbance of the supernatant was measured using a spectrophotometer (V-550, JASCO, Tokyo, Japan). Total chlorophyll $(\mathrm{a}+\mathrm{b})$ concentration was calculated as Chls $\mathrm{a}+\mathrm{b}(\mathrm{mg} / \mathrm{mL}$ in DMF) $=17.67 A_{646.8}+7.12 A_{663.8}$, where $A_{646.8}$ and $A_{663.8}$ are the absorbance at wavelengths of $646.8 \mathrm{~nm}$ and $663.8 \mathrm{~nm}$, respectively.

All the experiments were performed in triplicate. Although the results of representative single experiments are shown in Figures 2, 3, and 4, they were confirmed to be highly reproducible (there was less than $2.5 \%$ deviation in the results).

\section{Results}

\section{The spectrophotometer with special cuvette}

An apparatus for evaluating the photocatalyst activity of suspended fine particles was developed. It comprised a spectrophotometer equipped with a special cuvette. Figure 1 shows a schematic diagram of the system. The characteristics of this apparatus are as follows; (a) UV light (365 nm) was 


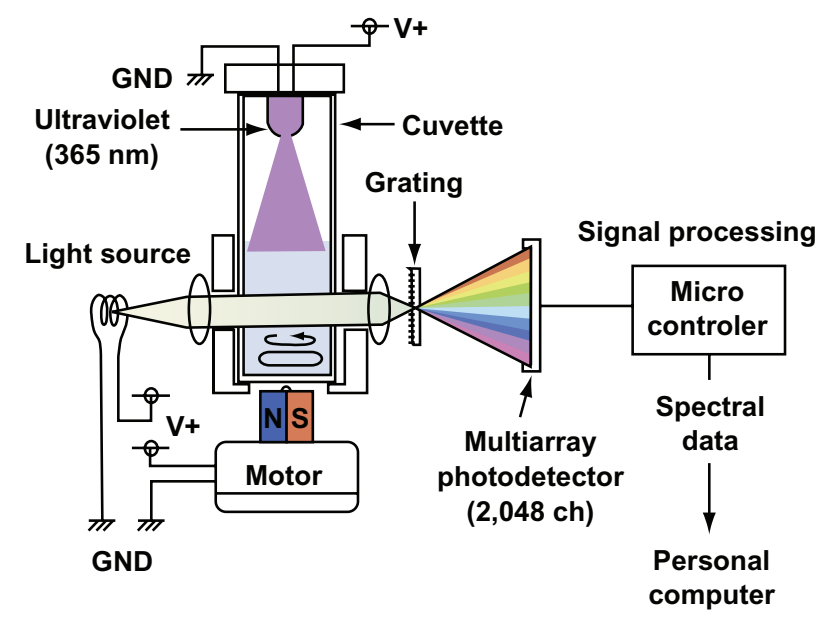

Figure I Schematic diagram of an apparatus for evaluating the photocatalytic activity of suspended fine particles in the liquid.

irradiated continuously at $0.7 \mathrm{~mW} / \mathrm{cm}^{2}$ in the surface of the sample by LED, which produces very low heat; (b) a magnetic stirrer is situated under the cuvette (diameter $=10 \mathrm{~mm}$, length $=43 \mathrm{~mm}$, working volume $=2.0 \mathrm{~mL}$, the temperature was kept at $25^{\circ} \mathrm{C}$ ) and the content can be mixed with a mini magnetic bar (diameter $=2 \mathrm{~mm}$, length $=5 \mathrm{~mm}$, the agitation speed $=600 \mathrm{rpm}$ ); (c) the absorbance and spectra (wavelength from $400 \mathrm{~nm}$ to $700 \mathrm{~nm}$ ) can be monitored continuously by means of personal computer. This apparatus enables the samples in the cuvette to be mixed homogeneously, giving a stable absorbance over a long period of time. It is therefore possible to obtain stable absorbance readings for even heterogeneous fine particles with high specific gravity (it was confirmed in various $\mathrm{TiO}_{2}$ solutions without $\mathrm{MB}$ under UV irradiation or no irradiation [data not shown]). The degradation of MB was used to evaluate photoactivity of $\mathrm{TiO}_{2}$. Changes in the spectra (400-700 nm) of photocatalytic decomposition of MB were monitored. It was observed that the absorbance decreased with

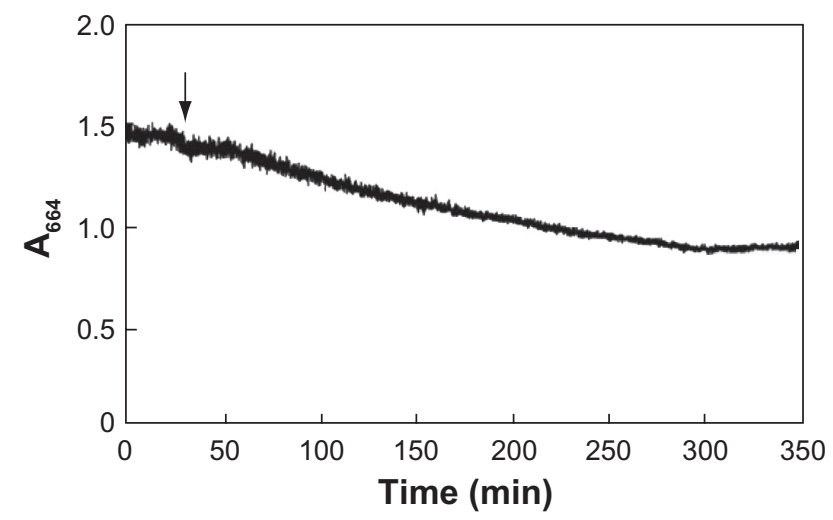

Figure 2 Changes in absorbance at $664 \mathrm{~nm}$ during photocatalytic reaction. ANA $\left(\mathrm{TiO}_{2}\right.$ anatase form) $125 \mathrm{mg} / \mathrm{L}$, methylene blue $5 \mathrm{mg} / \mathrm{L}$ in the distilled water at $25^{\circ} \mathrm{C}$. The arrow indicates the start of UV $(365 \mathrm{~nm})$ irradiation. time at each wavelength (data not shown). A wavelength of $664 \mathrm{~nm}$ was selected as the optimal wavelength for monitoring MB decomposition (Otsuka-Yao-Matsuo et al used the same wavelength for the measurement of MB decomposition). ${ }^{19}$ The absorbance at $664 \mathrm{~nm}$ of the sample solution $\left(\mathrm{MB}+\mathrm{TiO}_{2}\right)$ was not allowed to exceed to 1.9 , which was the limit for possible quantitative evaluation.

Figure 2 shows changes in the absorbance at $664 \mathrm{~nm}$ during photocatalytic decomposition of MB (5 mg/L) using ANA $(125 \mathrm{mg} / \mathrm{L})$. Before UV irradiation, the $A_{664}$ did not change. After UV irradiation, the $A_{664}$ decreased sharply due to photocatalytic decomposition of MB and then leveled off after about $300 \mathrm{~min}$. The above results showed that the process of photocatalytic decomposition of $\mathrm{MB}$ by $\mathrm{TiO}_{2}$ could be monitored optically, making it possible to use changes in the $A_{664}$ to measure the rate of reaction and to estimate the end of reaction.

Photocatalytic activities of $\mathrm{TiO}_{2}$ fine particles depend to a large extent on their crystalline form. In the case of the photoinactive RU (125 mg/L) or AM (125 mg/L) suspended in $\mathrm{MB}(5 \mathrm{mg} / \mathrm{L})$ solution at $25^{\circ} \mathrm{C}$, the $A_{664}$ did not change under UV irradiation (data not shown). Figure 3 shows the changes in $A_{664}$ during photocatalytic reaction with ANA and AP25. The photocatalytic activity of ANA in the distilled water is slightly higher than that of AP25. In artificial seawater, there was no photocatalytic activity of AP25, while the activity of ANA decreased significantly (Figure 3).

\section{Evaluation of the photocatalytic activities of various fine particles based on reaction rate constant}

The degradation of MB by photocatalysis is a first order reaction and can be expressed by Equation 1:

$$
\ln (A \mathrm{t}-A \mathrm{~s}) /\left(A_{0}-A \mathrm{~s}\right)=-k t
$$

where $t=$ time $(\min ), k=$ a constant $\left(\mathrm{min}^{-1}\right), A_{0}=$ the initial absorbance, $A \mathrm{t}=$ the absorbance at time $t$, and $A \mathrm{~s}=$ absorbance at the end of the process. The value of $\ln (A \mathrm{t}-A \mathrm{~s}) /$ $\left(A_{0}-A \mathrm{~s}\right)$ was plotted against time during the degradation of MB by photocatalysis (Figure 4). The major part of the reaction (about 70\%) can be described by simple first order kinetics. The rate constant $(k)$ was calculated from the initial straight line. Table 1 shows a comparison of the $k$ values for various photocatalysts. The $k$ values varied depending on the suspending liquid and on the type of photocatalysts. The photocatalytic activity of ANA in artificial seawater decreased 
A

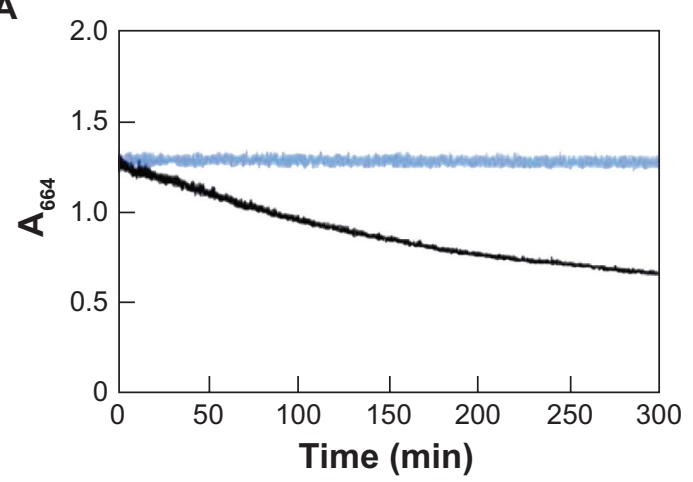

B

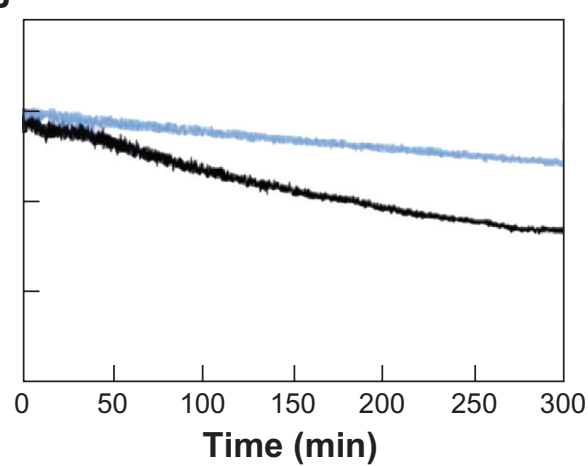

Figure 3 Changes in absorbance at $664 \mathrm{~nm}$ during photocatalytic reaction. A) ANA I $25 \mathrm{mg} / \mathrm{L}, \mathrm{MB} 5 \mathrm{mg} / \mathrm{L}$ at $\left.25^{\circ} \mathrm{C}, \mathrm{B}\right) \mathrm{AP} 25 \mathrm{I} 25 \mathrm{mg} / \mathrm{L}, \mathrm{MB} 5 \mathrm{mg} / \mathrm{L}$ at $25^{\circ} \mathrm{C}$. Black lines are in the distilled water, Blue lines: in artificial sea water.

to one third of that in ultrapure water. The $k$ value of STS-01 is 3.5 times larger than that of ANA or AP25. By using the values of $k$, it was possible to quantitatively evaluate and compare the photocatalytic activities of various fine particles.

\section{Effect of photocatalysis using various $\mathrm{TiO}_{2}$ fine particles on the physiological activities of $E$. gracilis}

The effect of photocatalysis using various $\mathrm{TiO}_{2}$ fine particles on the physiological activities of E. gracilis was investigated (Figure 5). The $k$ values of ANA (Figure 5C), AP25 (Figure 5D), and RU (Figure 5E) in the E. gracilis cell culture medium were determined on the basis of MB degradation by

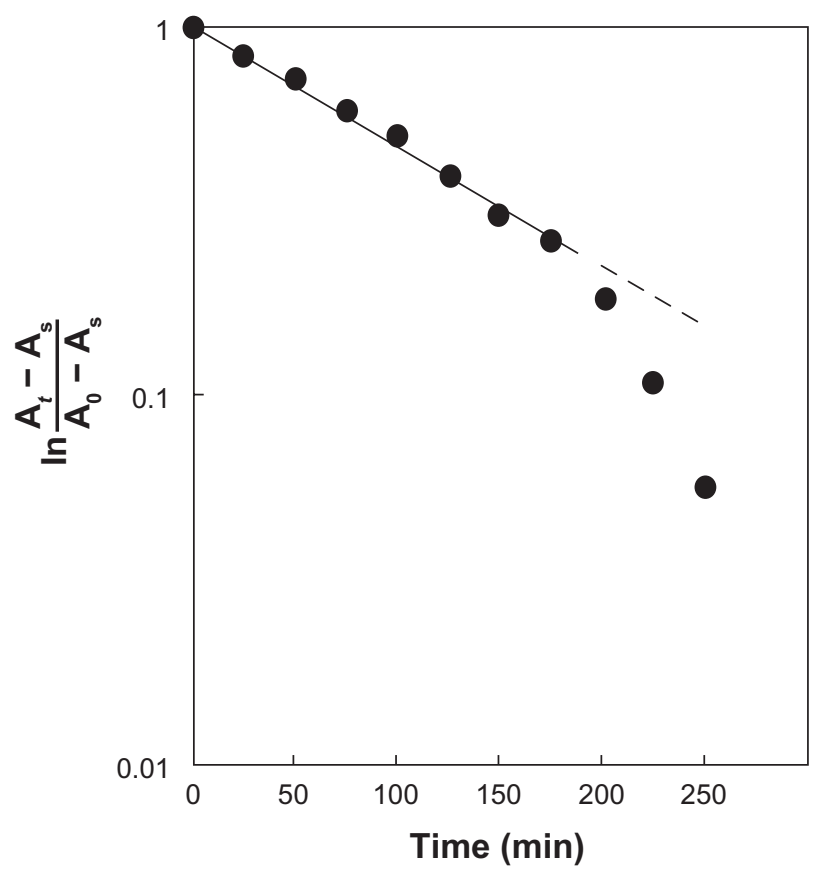

Figure 4 Logarithmic plot of $\ln \left(A_{t}-A_{s}\right) /\left(A_{0}-A_{s}\right)$ against time during photocatalytic reaction. Condition: ANA $125 \mathrm{mg} / \mathrm{L}, \mathrm{MB} 5 \mathrm{mg} / \mathrm{L}$ in the distilled water at $25^{\circ} \mathrm{C}$ (the data of Figure 2 was used). using the apparatus described earlier. The $k$ values of ANA, AP25, and RU in the medium were $7.1 \pm 0.09 \times 10^{-3}\left[\mathrm{~min}^{-1}\right]$, $5.1 \pm 0.07 \times 10^{-3}\left[\mathrm{~min}^{-1}\right]$, and $0\left[\mathrm{~min}^{-1}\right]$, respectively. For the distilled water, the $k$ value for ANA was $7.65 \pm 0.15 \times 10^{-3}$ $\left[\mathrm{min}^{-1}\right]$, for AP25, $8.05 \pm 0.12 \times 10^{-3}\left[\mathrm{~min}^{-1}\right]$, while for RU, $0\left[\mathrm{~min}^{-1}\right]$ as shown in Table 1 . The $k$ values decreased due to the salts in the medium.

E. gracilis was cultivated for 72 hours in a medium containing RU (Figure 5E). The cell growth, chlorophyll content, and alpha-tocopherol content were the same as those obtained in a medium without RU (Figure 5B). On the other hand, when ANA (Figure 5C) or AP25 (Figure 5D) was added to the medium, the cell growth was suppressed, chlorophyll content was the same, while alpha-tocopherol contents of E. gracilis were 1.3 and 1.2 times higher than that of the control, respectively.

\section{Discussion}

A spectrophotometer with special cuvette described here makes it possible to monitor continuously and the photocatalytic decomposition of MB by suspended fine particles using small amount of sample solution $(2.0 \mathrm{~mL})$

Table I $k$ values for various photocatalysts

\begin{tabular}{lll}
\hline Photocatalysts & Suspending liquid & $\mathbf{k} \times \mathbf{1 \mathbf { 0 } ^ { - 3 }}\left(\mathbf{m i n}^{-1}\right)$ \\
\hline ANA & distilled water & $7.65 \pm 0.15$ \\
AP25 & distilled water & $8.05 \pm 0.12$ \\
ANA & artificial seawater & $2.47 \pm 0.07$ \\
AP25 & artificial seawater & 0 \\
STS-01 & distilled water & $28.7 \pm 0.9$ \\
STS-02 & distilled water & $3.16 \pm 0.10$ \\
RU & distilled water & 0 \\
AM & distilled water & 0 \\
\hline
\end{tabular}

Notes: ANA: 100\% anatase crystalline form (Wako); AP25: 20\% rutile $80 \%$ anatase crystalline form (Japan Aerosil); STS-0 I: photocatalytic sol (Ishihara Sangyo); STS-02: photocatalytic sol (Ishihara Sangyo); RU: 100\% rutile crystalline form (Wako); AM: amorphous (Wako). Numbers represent mean value \pm SD. 


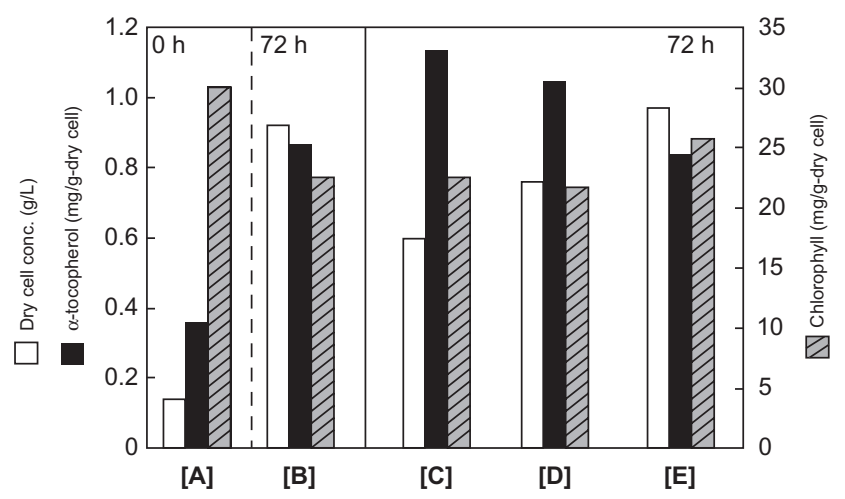

Figure 5 Effect of various photocatalysts on the physiological activities of E. gracilis at $72 \mathrm{~h}$ of cultivation. A) Inoculum at $0 \mathrm{~h}, \mathbf{B})$ no addition, C) $0.01 \%$ (w/v) ANA, D) $0.01 \%$ $(w / v)$ AP25, E) $0.01 \%(w / v)$ RU. The data are expressed as mean $\pm S D(n=3)$.

without sampling procedure. It was also possible to use changes in the absorbance to estimate the end of the reaction. In the case of sample containing heterogeneous fine particles in terms of size and specific gravity, it is difficult to evaluate the photocatalytic activity accurately (the results lack reproducibly). The method described here solves the problems, and it is excellent for measuring the photocatalytic activities of various fine particles accurately, reproducibly, and conveniently.

The changes in the absorbance varied depending on the type of photocatalysts and the suspending liquid. AP25 lost its photocatalytic activity, while that of ANA decreased significantly in artificial seawater (Figure 3). With this apparatus, it was possible to highlight the difference in the effects of seawater on ANA and AP25. It is clear that if ANA and AP25 are released into the sea, the environmental impact of ANA is more than that of AP25. It is known that the presence of inorganic anions such as chloride in water reduces the photocatalytic activity. This can be explained by the competition with dissolved organic carbons for adsorption or reaction..$^{20}$ It was reported that metal ions, (sodium and magnesium) resulted in enhanced photocatalytic activity at concentrations similar to those in seawater. ${ }^{21}$ The rate of photooxidative dye decolorization in seawater was slower than that in the $\mathrm{NaCl}$ solution even although sodium cation and chlorine anion are the major ions in seawater. ${ }^{21}$ The reasons for the differences observed between ANA and AP25 in artificial seawater is currently being investigated. By using the values of $k$ (reaction rate constant), it was possible to quantitatively evaluate and compare the photocatalytic activities of various fine particles under various conditions (Table 1). In the case of ANA, the photocatalytic activity in artificial seawater decreased to one third of that in distilled water. The photocatalytic activities of $\mathrm{TiO}_{2}$ fine particles differed greatly depending on such factors as the crystalline form, composition, particle size, and surface area. ${ }^{8,13}$

When ANA and AP25 were added to the medium under illumination by black light, the growth of E. gracilis was suppressed but their alpha-tocopherol contents increased (Figure 5). The effects of ANA on E. gracilis were more pronounced than those of AP25. This may be due to the fact that the $k$ value of ANA in the E. gracilis cell culture medium was higher than that of AP25. The modified Hutner medium contains many kinds of salts and this resulted in decrease in the $k$ values of ANA and AP25. This is consistent with the results obtained when they were suspended in artificial seawater (Figure 3). It is well known that various kinds of ROS are produced on $\mathrm{TiO}_{2}$ surface exposed to light rays of wavelengths below $410 \mathrm{~nm}$. Among these, hydroxyl radical is dominant and is known to be responsible for the oxidative stress on living cells. ${ }^{10}$ The ROS produced by photocatalytic reaction enhanced the alpha-tocopherol production by E. gracilis. This prevents oxidative decomposition of chlorophyll, lipid membranes, and proteins by ROS. ${ }^{22}$ through scavenging ROS released during oxidative stress. ${ }^{23}$ It was reported that the antioxidant alpha-tocopherol synthesis was activated to prevent damage by ROS..$^{24}$ It has also been reported that antioxidant carotenoid biosynthesis is regulated by ROS in some microalgae. ${ }^{25}$ The increase in alpha-tocopherol content of E. gracilis exposed to $\mathrm{TiO}_{2}$ suggests that the $\mathrm{TiO}_{2}$ fine particle promoted production of alpha-tocopherol and/or selected E. gracilis cells with high content of antioxidant. Thus, $\mathrm{TiO}_{2}$ may be used to enhance production of useful antioxidants.

Quantitative evaluation of photocatalytic activities of fine particles using the apparatus developed in this study is very important, considering the ecotoxicity and environmental impacts of these particles. By knowing the potential risks of these photocatalytic particles, it is possible to make a risk-benefit analysis.

\section{Acknowledgment}

This study was supported in part by a Grant-in-Aid for Exploratory Research (nr 20651006) from the Japan Society for the Promotion of Science (JSPS).

\section{Disclosure}

The authors report no conflicts of interest in relation to this paper. 


\section{References}

1. Nagasawa H, Ichimura A, Isoda S. Carboxylate-passivated silver nanoparticles and their application to sintered interconnection: a replacement for high temperature lead-rich solders. J Electron Mater. 2010;39:1233-1240.

2. Liu SQ, Tang ZY. Nanoparticle assemblies for biological and chemical sensing. J Mater Chem. 2010;20:24-35.

3. Narayanan R. Recent advances in noble metal nanocatalysts for Suzuki and Heck cross-coupling reactions. Molecules. 2010;15:2124-2138.

4. Ogura H, Maruyama M, Matsubayashi R, et al. Nanoscale particle therapies for wounds and ulcers. Nanomedicine. 2010;5:641-656.

5. Millis A, Lepre A, Elliott N, Bhopal S, Parkin I, O’Neill S. Characterisation of the photocatalyst Pilkington Active $^{\mathrm{TM}}$ : a reference film photocatalyst? J Photochem Photobiol A Chem. 2004;160:213-224.

6. Esterkin CR, Negro AC, Alfano OM, Cassano AE. Air pollution remediation in a fixed bed photocatalytic reactor coated with $\mathrm{TiO}_{2}$. AIChEJ. 2005;51:2298-2310.

7. Choi H, Stathatos E, Dionysiou DD. Sol-gel preparation of mesoporous photocatalytic $\mathrm{TiO}_{2}$ films and $\mathrm{TiO}_{2} / \mathrm{Al}_{2} \mathrm{O}_{3}$ composite membranes for environmental applications. Appl Catal B Environ. 2006;63:60-67.

8. Adams LK, Lyon DY, Alvarez PJJ. Comparative eco-toxicity of nanoscale $\mathrm{TiO}_{2}, \mathrm{SiO}_{2}$, and $\mathrm{ZnO}$ water suspension. Water Res. 2006;40:3527-3532.

9. Kaida T, Kobayashi K, Adachi M, Suzuki F. Optical characteristics of titanium oxide interference film and the film laminated with oxides and their applications for cosmetics. J Cosmet Sci. 2004;55:219-220.

10. Cai R, Kubota Y, Shuin T, et al. Induction of cytotoxicity by photoexcited $\mathrm{TiO}_{2}$ particles. Cancer Res. 1992;52:2345-2348.

11. Otaki M, Hirata T, Ohgaki S. Aqueous microorganisms inactivation by photocatalytic reaction. Water Sci Technol. 2000;43:115-118.

12. Sunada K, Watanabe T, Hashimoto K. Studies on photokilling of bacteria $\mathrm{TiO}_{2}$ thin film. J Photochem Photobiol A Chem. 2003;156:227-233.

13. Hund-Rinke K, Simon M. Ecotoxic effect of photocatalytic active nanoparticles $\left(\mathrm{TiO}_{2}\right)$ on algae and daphnids. Environ Sci Pollut Res. 2006; 13:225-232.

14. Oberdorster G, Oberdorster E, Oberdorster J. Nanotoxicology: an emerging discipline evolving from studies of ultrafine particles. Environ Health Perspect. 2005;113:823-839.
15. Xu NP, Shi ZF, Fan YQ, Dong JH, Shi J, Hu MZC. Effects of particle size of $\mathrm{TiO}_{2}$ on photocatalytic degradation of methylene blue in aqueous suspensions. Ind Eng Chem Res. 1999;38:373-379.

16. Ogbonna JC, Tanaka H. Cyclic autotrophic/heterotrophic cultivation of photosynthetic cells: a method of achieving continuous cell growth under light/dark cycles. Bioresour Technol. 1998;65:65-72.

17. Shigeoka S, Onishi T, Nakano Y, Kitaoka S. The contents and subcellular distribution of tocopherols in Euglena gracilis. Agric Biol Chem. 1986;50:1063-1065.

18. Porra RJ, Thompson WA, Kriedemann PE. Determination of accurate extinction coefficients and simultaneous equations for assaying chlorophylls $a$ and $b$ extracted with four different solvents: verification of the concentration of chlorophyll standards by atomic absorption spectroscopy. Biochim Biophys Acta. 1989;975:384-394.

19. Otsuka-Yao-Matsuo S, Omata T, Yoshimura M. Photocatalytic behavior of cerium titanates, $\mathrm{CeTiO}_{4}$ and $\mathrm{CeTi}_{2} \mathrm{O}_{6}$ and their composite powders with $\mathrm{SrTiO}_{3}$. J Alloys Compd. 2004;376:262-267.

20. Chen HY, Zahraa O, Bouchy M. Inhibition of the adsorption and photocatalytic degradation of an organic contaminant in an aqueous suspension of $\mathrm{TiO}_{2}$ by inorganic ions. J Photochem Photobiol A Chem. 1997; 108:37-44.

21. Makita M, Harata A. Photocatalytic decolorization of rhodamine B dye as a model of dissolved organic compounds: Influence of dissolved inorganic chloride salts in seawater of the sea of Japan. Chem Eng Process. 2008;47:859-863.

22. Tani Y, Tsumura H. Screening for tocopherol-producing microorganisms and alpha-tocopherol production by Euglena gracilis Z. Agric Biol Chem. 1989;53:305-312.

23. Munne-Bosch S, Alegre L. The function of tocopherols and tocotrienols in plants. CRC Crit Rev Plant Sci. 2002;21:31-57.

24. Fujita T, Ogbonna JC, Tanaka H, Aoyagi H. Effects of reactive oxygen species on alpha-tocopherol production in mitochondria and chloroplasts of Euglena gracilis. J Appl Phycol. 2009;21:185-191.

25. Kobayashi M, Kakizono T, Nagai S. Enhanced carotenoid biosynthesis by oxidative stress in acetate induced cyst cells of a green unicellular alga, Haematococcus pluvialis. Appl Microbiol Biotech. 1993;59:867-873.
Nanotechnology, Science and Applications

\section{Publish your work in this journal}

Nanotechnology, Science and Applications is an international, peerreviewed, open access journal that focuses on the science of nanotechnology in a wide range of industrial and academic applications. It is characterized by the rapid reporting across all sectors, including engineering, optics, bio-medicine, cosmetics, textiles, resource sustainability

\section{Dovepress}

and science. Applied research into nano-materials, particles, nanostructures and fabrication, diagnostics and analytics, drug delivery and toxicology constitute the primary direction of the journal. The manuscript management system is completely online and includes a very quick and fair peer-review system, which is all easy to use. 